\title{
THE DISCOVERY OF AN X-RAY/UV STELLAR FLARE FROM THE LATE-K/EARLY-M DWARF LMC 335
}

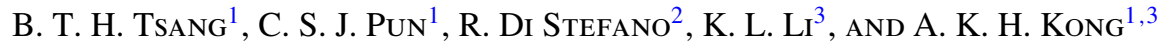 \\ ${ }^{1}$ Department of Physics, The University of Hong Kong, Pokfulam Road, Hong Kong \\ 2 Harvard-Smithsonian Center for Astrophysics, 60 Garden Street, Cambridge, MA 02138, USA \\ ${ }^{3}$ Institute of Astronomy and Department of Physics, National Tsing Hua University, Hsinchu, Taiwan \\ Received 2012 March 15; accepted 2012 May 25; published 2012 July 13
}

\begin{abstract}
We report the discovery of an X-ray/UV stellar flare from the source LMC 335, captured by XMM-Newton in the field of the Large Magellanic Cloud. The flare event was recorded continuously in X-ray for its first $10 \mathrm{hr}$ from the precursor to the late decay phases. The observed fluxes increased by more than two orders of magnitude at its peak in X-ray and at least one in the UV as compared to quiescence. The peak 0.1-7.0 keV X-ray flux is derived from the two-temperature APEC model to be $\sim(8.4 \pm 0.6) \times 10^{-12} \mathrm{erg} \mathrm{cm}^{-2} \mathrm{~s}^{-1}$. Combining astrometric information from multiple X-ray observations in the quiescent and flare states, we identify the NIR counterpart of LMC 335 as the Two Micron All Sky Survey source J05414534-6921512. The NIR color relations and spectroscopic parallax characterize the source as a Galactic K7-M4 dwarf at a foreground distance of (100-264) pc, implying a total energy output of the entire event of $\sim(0.4-2.9) \times 10^{35} \mathrm{erg}$. This report comprises detailed analyses of this late-K/early-M dwarf flare event that has the longest time coverage yet reported in the literature. The flare decay can be modeled with two exponential components with timescales of $\sim 28$ minutes and $\sim 4 \mathrm{hr}$, with a single-component decay firmly ruled out. The X-ray spectra during flare can be described by two components, a dominant high-temperature component of $\sim 40-60 \mathrm{MK}$ and a low-temperature component of $\sim 10 \mathrm{MK}$, with a flare loop length of about 1.1-1.3 stellar radius.
\end{abstract}

Key words: stars: activity - stars: coronae - stars: flare - stars: individual (LMC 335) - stars: late-type - X-rays: stars

Online-only material: color figures

\section{INTRODUCTION}

Stellar flares from magnetically active stars provide useful tools to diagnose the physical processes governing the observed multi-wavelength characteristics, along with the physical and chemical evolutions of stellar coronae (Benz \& Güdel 2010). Stars of spectral types F, G, K, and M are found to exhibit flare activities of various forms and scales. Dwarf flares have been extensively studied at wavelengths spanning from radio to $\mathrm{X}$-ray, with UV and X-ray observations particularly effective for investigations of the cool dwarfs due to their characteristic ranges of coronal temperatures during flare (Osten et al. 2006; Berger et al. 2010). Many studies of flare stars have focused on the derivation of information about the magnetic activities associated with spatially unresolved flare events. The immense bursts of energy from stellar flares are believed to be generated from dynamo processes persistent in the stellar interiors and atmospheres. Hydrodynamic simulations (Reale et al. 1997; Favata et al. 2001; Allred et al. 2006) and the quasi-static radiative cooling models (van den Oord \& Mewe 1989; Tsuboi et al. 2000) had been widely adopted to study the stellar magnetic activities based on their flare profiles.

Following the early detections of a small sample of flare candidates such as AT Mic and AD Leo (Kahn et al. 1979) with the HEAO-1 satellite, the Einstein and EXOSAT observatories extended the study to cover X-ray emission from stellar flares with better sensitivity and longer time coverage (Pallavicini et al. 1990). The observed energetic X-ray flares were divided into two subclasses based on the timescales of the flaring profiles: "impulsive" flares with decay times of up to tens of minutes are believed to originate from relatively compact loops similar to those found on the Sun and "long-decay" flares with typical hours-long decay time observed in solar two-ribbon flares (Priest \& Forbes 2002). Pallavicini et al. (1990) observed 25 K5 to M7 stars within $25 \mathrm{pc}$ with quiescent $(0.05-2 \mathrm{keV})$ luminosities $\log L\left(\mathrm{erg} \mathrm{s}^{-1}\right)$ of $\sim 27$ to $\sim 30$, and found X-ray flares with energies from $\sim 3 \times 10^{30}$ to $1 \times 10^{34} \mathrm{erg}$, and flare temperatures of $\sim 20-40 \mathrm{MK}$. These values are comparable to those obtained from Einstein and these observations have extended the studies on stellar flares with improved sensitivity especially for their quiescent emissions.

In the extreme-UV (EUV) wavelength, Mullan et al. (2006) conducted a survey on 44 type $\mathrm{F}$ to $\mathrm{M}$ stars in the Extreme UltraViolet Explorer (EUVE) database containing a total of 134 flares to explore the underlying dynamo mechanisms using physical parameters of the flare loops derived from a homogeneous set of flare light curves. The primary parameters investigated are the flare loop length and the magnetic fields during flares. It was suggested that the observed increase of maximum flare loop sizes from the warmer K2 to M0 sample to the cooler and later ones could be due to a transition from a core-convection dynamo to a turbulent dynamo.

After scattered Einstein and EXOSAT observations, the Röntgen Satellite (ROSAT) All-Sky Survey (RASS) provided a comprehensive coverage for the entire sky in soft X-ray. Tsikoudi et al. (2000) selected a sample of 14 active stars from K0 to M5.5 in the RASS database to search for concurrent energetic flares in both EUV and X-ray energies. Due to the observing strategy in the scanning mode, all the detected flares are of longer duration with event ages longer than an hour. High correlations of the X-ray $(0.1-2.4 \mathrm{keV})$ and EUV $(60-140 \AA$ or 110-200 $\AA$ ) luminosities could be deduced for all observed flares, with flare luminosities $\log L$ ( $\mathrm{erg} \mathrm{s}^{-1}$ ) from 27.3 to 30.0 for X-ray and 26.6 to 29.7 in EUV. 
Table 1

X-ray Detection of LMC 335 by the XMM-Newton EPIC

\begin{tabular}{|c|c|c|c|c|c|c|c|c|}
\hline ObsID & Det-ExpID & $\begin{array}{c}\text { Date } \\
(\mathrm{MM} / \mathrm{DD} / \mathrm{YYYY})\end{array}$ & $\begin{array}{c}\text { Start Time } \\
\text { (UT HH:MM) }\end{array}$ & $\begin{array}{l}\text { Exp. Time } \\
\text { (ks) }\end{array}$ & Mode $^{\mathrm{a}}$ & Opt. Fil. & Det. ML & $\begin{array}{l}\text { Average Count Rate } \\
\qquad\left(\mathrm{s}^{-1}\right)\end{array}$ \\
\hline 0113000401 & M2S007 & $05 / 23 / 2001$ & $07: 33$ & 47.8 & PFW & Thick & 24.4 & 0.0026 \\
\hline 0117510201 & PNS010 & $02 / 11 / 2000$ & 09:05 & 10.8 & PFW & Medium & 55.2 & 0.0172 \\
\hline 0117730501 & M1S014 & $02 / 17 / 2000$ & $13: 29$ & 10.2 & PFW & Medium & 20.8 & 0.0064 \\
\hline 0117730501 & M1S016 & $02 / 17 / 2000$ & $17: 20$ & 14.2 & PPW2 & Medium & 24.1 & 0.0051 \\
\hline 0125120101 & M1S008 & $05 / 26 / 2000$ & $12: 54$ & 31.9 & PFW & Medium & 28.7 & 0.0034 \\
\hline 0125120101 & M2S010 & $05 / 26 / 2000$ & $12: 54$ & 31.9 & PFW & Medium & 27.8 & 0.0035 \\
\hline 0125120101 & $\mathrm{PNS} 006^{\mathrm{b}}$ & $05 / 26 / 2000$ & $11: 03$ & 38.6 & PFW & Medium & 170.6 & 0.0159 \\
\hline 0125120201 & $\mathrm{M} 1 \mathrm{~S} 007^{\mathrm{c}}$ & $05 / 27 / 2000$ & 01:50 & 40.2 & FastUC & Medium & 6056.0 & 0.1218 \\
\hline 0125120201 & $\mathrm{M} 2 \mathrm{~S} 009^{\mathrm{c}}$ & $05 / 27 / 2000$ & $01: 50$ & 40.2 & FastUC & Medium & 6055.7 & 0.1193 \\
\hline 0413180201 & M2S002 & $10 / 02 / 2006$ & 01:01 & 13.6 & FastUC & Medium & 9.0 & 0.0027 \\
\hline 0413180301 & $\mathrm{M} 2 \mathrm{~S} 002$ & $10 / 02 / 2006$ & $18: 36$ & 16.2 & FastUC & Medium & 15.5 & 0.0033 \\
\hline
\end{tabular}

Notes.

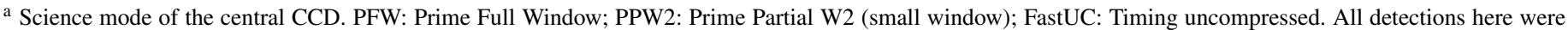
made with Imaging mode data.

${ }^{\mathrm{b}} X M M$ PN Observations capturing the quiescence, used for spectral analysis of quiescence (Q) phase (Table 5).

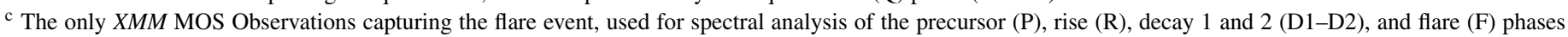
(Table 5).

The better spatial and spectral resolution of the XMM-Newton and Chandra X-ray observatories vastly enhance the investigations of flare stars especially in the lower temperature end. Flare emissions from a number of dwarfs later than M8 were observed (Rutledge et al. 2000; Stelzer et al. 2006; Robrade \& Schmitt 2009; Robrade et al. 2010; Gupta et al. 2011). These observations also enabled quiescent emission of late-type stars to be detected and studied at much fainter limits, revealing the highly unstable nature during quiescence, commonly referred to as quasi-quiescence states. Simultaneous monitoring in the $\mathrm{X}$-ray and optical/UV wavelengths by XMM-Newton provides further constraints on the possible emission mechanisms associated with the magnetic activities. The availability of highresolution X-ray spectroscopy supports the studies of coronal elemental abundances (Audard et al. 2003; Sanz-Forcada et al. 2004) as well as the construction of emission measure distribution during different stages of the flare evolution for detailed investigations of the temperature structure of the stellar coronae (Huenemoerder et al. 2001; Raassen et al. 2003).

The discovery of a previously unclassified flare object in the field of LMC by XMM-Newton, first detected in the quiescent state by the ROSAT High Resolution Imager (HRI) survey (Sasaki et al. 2000), is reported in this paper. The detected $\mathrm{X}$-ray flare is found to be positioned at $\sim 2$ ". 5 from the cataloged position of source 335 (Sasaki et al. 2000), hereafter referred to as LMC 335. In addition to ROSAT and XMM-Newton, the source was also observed in X-ray quiescence with the Chandra and Swift satellites. In Section 2, the X-ray flare and quiescent observations of LMC 335, along with UV and optical images of the field, are described. Results of the findings are presented in Section 3: with Section 3.1 focusing on the source and counterpart identification, and Sections 3.2 and 3.3 on the timing and spectral analysis, respectively. Discussions and conclusions are presented in Section 4.

\section{OBSERVATIONS AND DATA ANALYSIS}

After the first detection by the ROSAT HRI, LMC 335 had been captured in X-ray with various instruments including XMM-Newton, Chandra, and Swift. Only one of the $X M M-N e w t o n$ observations captured an X-ray/UV flare event, while all the other observations were made during quiescent states. Observational data in X-ray were obtained from the High Energy Astrophysics Science Archive Research Center (HEASARC) database. In this section, we summarize the multiwavelength observations of LMC 335 and highlight those related to the flare event.

In Table 1, we list all the XMM-Newton European Photon Imaging Camera (EPIC) observations that included LMC 335. These observations were originally targeted at the pulsar PSR0540-69 and thus LMC 335 was at an off-axis angle of $\sim 9^{\prime}$. The analysis was performed using the standard XMM-Newton Science Analysis System (SAS) version 11.0.0. With a majority of the quiescent state and flaring state X-ray photons contained in the lower energy bins, the energy band used for exposure map corrections and source detection was limited to $0.1-7.0 \mathrm{keV}$. At the position of LMC 335, source spectra were extracted with the SAS task evselect using a circular region of radius $10^{\prime \prime}$, while the background was measured from a nearby source-free circular region of radius $40^{\prime \prime}$ on the same $\mathrm{CCD}$. The same regions were adopted to generate light curves, with the task epiclccoor used to perform the background subtraction. All the spectra and response files were grouped with minimum counts of 20 and 10 for the flare duration and the quiescent phase, respectively. The grouped spectra were then analyzed with XSPEC version 12.7.0.

The X-ray flare event from LMC 335 was recorded by the two $X M M-N e w t o n$ EPIC MOS cameras in medium optical filters on 2000 May 27 during a 40 ks observation (ObsID 0125120201 in Table 1). During the observation, while the central CCDs (CCD 1) of the two MOS cameras were operating in the Timing mode, the outer CCDs (CCD 2-7), where LMC 335 was captured, were in the Imaging mode. Unfortunately, the more sensitive EPIC PN camera was in the Small Window mode and did not include LMC 335 in its field of view. Therefore, only data from two MOS cameras are available for the X-ray flare analysis.

In addition to the EPIC cameras, the Optical Monitor (OM) on board XMM-Newton also recorded simultaneous UV broadband observations of LMC 335. Three of the 10 $X M M$-Newton observations have $\mathrm{OM}$ imaging coverage, one of which was fortuitously taken during the flare. During the 
Table 2

UV Detection of LMC 335 by the XMM-Newton Optical Monitor

\begin{tabular}{|c|c|c|c|c|c|c|c|}
\hline OM Exp. ID & UV Filter & $\begin{array}{c}\text { Start Time } \\
\text { (UT HH:MM) }\end{array}$ & $\begin{array}{l}\text { Exp. Time } \\
\text { (s) }\end{array}$ & $\begin{array}{l}\text { Count Rate } \\
\left(\text { counts s }^{-1}\right)\end{array}$ & Magnitude & $\begin{array}{c}\text { Flux } \\
\left(10^{-16} \mathrm{erg} \mathrm{cm}^{-2} \mathrm{~s}^{-1} \AA^{-1}\right)\end{array}$ & $\begin{array}{l}\text { Luminosity }^{\mathrm{a}} \\
\left(10^{30} \mathrm{erg} \mathrm{s}^{-1}\right)\end{array}$ \\
\hline \multicolumn{8}{|c|}{0125120101 on 2000 May 26} \\
\hline 440 & $U V W 2$ & $16: 40$ & 4000 & $\leqslant 0.07$ & $\geqslant 17.7$ & $\leqslant 4.2$ & $\leqslant 2.3$ \\
\hline \multicolumn{8}{|c|}{0125120201 on 2000 May 27} \\
\hline 432 & $U V W 1$ & $02: 18$ & 1000 & $\leqslant 0.38$ & $\geqslant 18.3$ & $\leqslant 1.8$ & $\leqslant 1.4$ \\
\hline $436^{\dagger}$ & $U V W 1$ & 04:09 & 1000 & $3.81 \pm 0.09$ & $15.75 \pm 0.03$ & $(18.0 \pm 0.5)$ & $(2.5-17.4)$ \\
\hline 440 & UVM2 & $07: 39$ & 2500 & $\leqslant 0.13$ & $\geqslant 18.0$ & $\leqslant 2.6$ & $\leqslant 1.2$ \\
\hline 444 & $U V W 2$ & $13: 14$ & 4000 & $\leqslant 0.08$ & $\geqslant 17.6$ & $\leqslant 4.4$ & $\leqslant 1.9$ \\
\hline 448 & $U V W 2$ & $18: 44$ & 2500 & $\leqslant 0.09$ & $\geqslant 17.5$ & $\leqslant 4.7$ & $\leqslant 2.0$ \\
\hline \multicolumn{8}{|c|}{011300401 on 2001 May 23} \\
\hline 401 & UVM2 & $08: 34$ & 3500 & $\leqslant 0.12$ & $\geqslant 18.1$ & $\leqslant 2.6$ & $\leqslant 1.5$ \\
\hline 404 & UVM2 & $11: 45$ & 3500 & $\leqslant 0.11$ & $\geqslant 18.2$ & $\leqslant 2.3$ & $\leqslant 1.4$ \\
\hline 405 & $U V W 2$ & $13: 53$ & 3500 & $\leqslant 0.08$ & $\geqslant 17.6$ & $\leqslant 4.6$ & $\leqslant 2.5$ \\
\hline 408 & $U V W 2$ & 17:04 & 3500 & $\leqslant 0.07$ & $\geqslant 17.7$ & $\leqslant 4.2$ & $\leqslant 2.3$ \\
\hline
\end{tabular}

Notes. $3 \sigma$ upper limits were computed for non-detections.

$\dagger$ The only OM exposure in which LMC 335 was detected.

a The luminosity range is obtained by the assumed distance range of (100-264 pc), while the upper limits assumed the distance 264 pc.

flaring observation, the OM took a series of UV images with filters $U V W 1(245-320 \mathrm{~nm}), U V M 2(205-245 \mathrm{~nm})$, and $U V W 2$ (180-225 nm), with exposure time ranging from $1 \mathrm{ks}$ to $4 \mathrm{ks}$. The exposures were all taken with the lateral Window 1 with $2 \times 2$ pixel binning. Details of the OM observations are summarized in Table 2. LMC 335 was not detected by the OM in the UV except for one $U V W 1$ observation taken roughly $0.5 \mathrm{hr}$ after the X-ray flux maximum. For the non-detections, the $3 \sigma$ upper limits of count rates, magnitudes, and fluxes were estimated with the average count rate computed from five sourcefree background regions near the source position. The detected magnitude was taken from the omichain-generated Observation Window source lists. The magnitude to UV flux and upper limits conversion were done using the standard reference fluxes of Vega.

X-ray emission from the quiescent phase of LMC 335 was independently detected by the Chandra and Swift satellites in addition to XMM-Newton. In the case of Chandra, three High Resolution Camera-I (HRC-I) non-grating observations of LMC 335 were taken in three separate pointings on 1999 August 31 (ObsID 132) and 2000 June 21 (ObsIDs 1735 and 1736). The event lists were merged using the Chandra Interactive Analysis of Observations version 4.2 task dmmerge with an effective exposure time of $\sim 48 \mathrm{ks}$. The observed quiescent count rate of $(3.4 \pm 0.5) \times 10^{-3} \mathrm{~s}^{-1}$ was consistent with that measured by the $X M M-N e w t o n$. On the other hand, the better angular resolution of the HRC data $\left(<0^{\prime \prime} .5\right)$ provides more accurate position than the XMM-Newton MOS data (2".0).

The field of LMC 335 had been imaged by Swift in a series of observations targeted also at PSR0540-69. A total of 22 sets of data were obtained spanning from 2005 April to 2010 November with an integrated exposure time of $\sim 170 \mathrm{ks}$. Similar to the Chandra observations, the data were taken when LMC 335 was in quiescent states and the $\mathrm{X}$-ray emission was weak, with count rate of $(1.1 \pm 0.2) \times 10^{-3}$ counts $^{-1}$. The event lists were combined before light curves and spectra were extracted with the Swift tool xrtgrblcspec. A low signal-to-noise spectrum could be extracted from the combined event list, and was found to be consistent with (but of poorer quality than) the $X M M-N e w t o n$ data. The X-ray position and positional errors were computed using the task xrtcentroid for comparison with the other observations.

In addition to X-ray observations, archival optical/NIR data taken with European Southern Observatory Wide Field Imager (ESO-WFI) of LMC 335 are available through the observations of the association LH 104. The raw ESO-WFI images taken on 2004 November 21 were obtained from the ESO Archive ${ }^{4}$ in four filters, $B$ Bessel (ESO\# 878), Rc/ 162 (ESO\# 844), Z+60 (ESO\# 846), and H_alpha 7 (ESO\# 856), with cumulative exposure time of about $4,2,43$, and 25 minutes, respectively. With the reduced images, astrometry was done by registering five Two Micron All Sky Survey (2MASS) and one Tycho bright stars near the field of LMC 335 with no known proper motions. The errors of the World Coordinate System mapping for all the ESO-WFI images are well below 0 '. 1 near the source LMC 335. Two cataloged sources, 2MASS J05414534-6921512 (Skrutskie et al. 2006) and DENIS J054145.2-692151 (DENIS Consortium 2005), are found to be closest to the position of LMC 335 and are considered to be the IR counterparts of the source. Since there is no systematic differences in the $J$ - and $K_{S}$-band measurements between 2MASS and DENIS (Cabrera-Lavers \& Garzón 2003), the consistent $J$ - and $K_{S}$-band magnitudes together with the slight positional offset of $\sim 0^{\prime} .4$ of these two cataloged sources suggest that these counterparts are indeed the same physical object.

\section{RESULTS}

\subsection{Source Position and Near-IR Counterpart}

Identification of counterparts of LMC 335 could reveal the nature of the X-ray flare event. Positions of the source computed from all the X-ray and IR data are listed with the $1 \sigma$ errors in Table 3. For the Chandra HRC data, the reported position for the source at quiescent state was obtained by the result obtained from image binning factors of 16. In the case of XMMNewton, both the flare and quiescent state positions are reported. The two MOS flare detections were used to determine the flare state position while all other detections were used for quiescent

\footnotetext{
4 http://archive.eso.org/eso/eso_archive_main.html
} 
Table 3

Source Positions of LMC 335 Derived from X-ray and NIR Instruments

\begin{tabular}{|c|c|c|c|c|c|}
\hline Instrument & Detector & $\begin{array}{c}\text { R.A. } \\
(\mathrm{J} 2000.0)\end{array}$ & $\begin{array}{l}\text { R.A. Error } \\
\text { (arcsec) }\end{array}$ & $\begin{array}{c}\text { Decl. } \\
(\mathrm{J} 2000.0)\end{array}$ & $\begin{array}{c}\text { Decl. Error } \\
(\operatorname{arcsec})\end{array}$ \\
\hline \multicolumn{6}{|c|}{ X-ray Positions } \\
\hline Chandra & HRC-I & 054145.28 & 1.09 & -692151.5 & 1.0 \\
\hline XMM-Newton (Flare) & MOS & 054145.42 & 1.42 & -692149.4 & 1.4 \\
\hline XMM-Newton (Quiescent) & $\mathrm{MOS}+\mathrm{PN}$ & 054145.29 & 0.86 & -692150.6 & 0.9 \\
\hline Swfit & XRT-PC & 054145.35 & 3.60 & -692151.2 & 3.6 \\
\hline$R O S A T$ & HRI & 054144.60 & 4.40 & -692151.1 & 4.4 \\
\hline \multicolumn{6}{|c|}{ NIR Positions } \\
\hline \multirow{2}{*}{\multicolumn{2}{|c|}{$\begin{array}{l}\text { 2MASS } \\
\text { DENIS }\end{array}$}} & 054145.34 & 0.19 & -692151.22 & 0.1 \\
\hline & & 054145.27 & $\leqslant 1$ & -692151.10 & $\leqslant 1$ \\
\hline
\end{tabular}

Note. NIR counterparts are 2MASS J05414534-6921512 and DENIS J054145.2-692151.

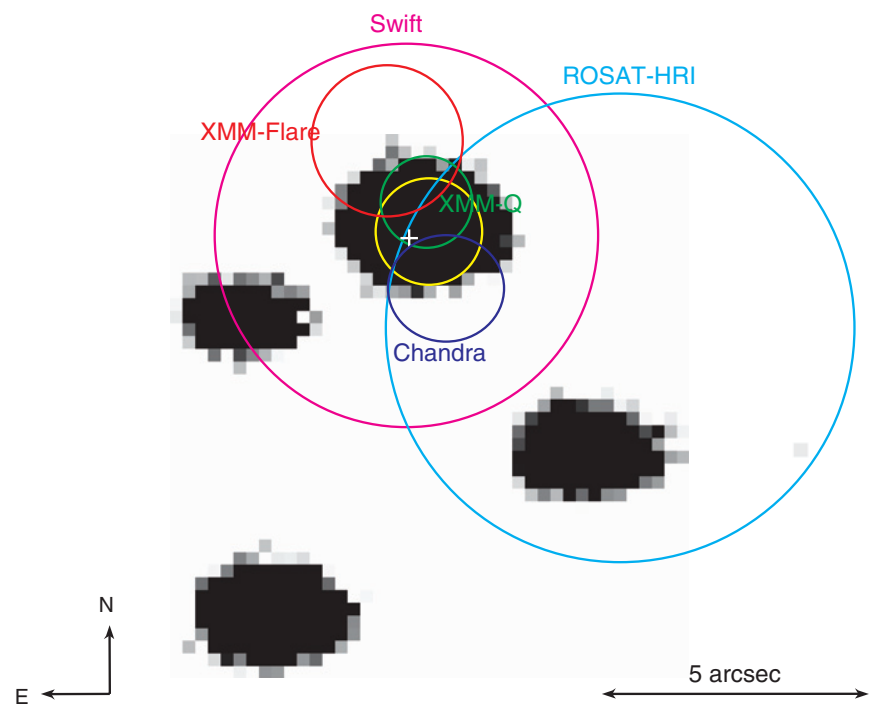

Figure 1. ESO WFI $R_{c}$-band image of the LMC 335 field shown with NIR and X-ray source positions and $1 \sigma$ error circles. White cross: 2MASS J05414534-6921512 counterpart, yellow circle: DENIS J054145.2-692151 counterpart, red circle: weighted average position from XMM-Newton flare observations, green circle: weighted average position from the XMM-Newton quiescent observations, blue circle: Chandra HRC position, cyan circle: ROSAT HRI position, magenta circle: Swift X-Ray Telescope (XRT) position.

(A color version of this figure is available in the online journal.)

state measurement. Before weight averaging the results from the individual detections, the $1 \sigma$ positional errors were obtained by combining in quadrature the statistical maximum likelihood (ML) fitting errors with the systematic error of the pointing of XMM-Newton, taken to be 2".0 (Guainazzi 2011) in this work. A similar approach of positional error calculation was adopted for the XMM-Newton Serendipitous source catalog (Watson et al. 2009). With the best angular resolution, the Chandra HRC data provide the smallest uncertainty in the X-ray position of the source at its quiescent state, despite the low count rate recorded for the data. On the contrary, the low resolution of Swift and ROSAT data imply poor astrometry. The $1 \sigma$ uncertainty contours of the quiescent and flare states' X-ray emission are overlaid on the ESO-WFI $R c$-band image of the field in Figure 1, along with the cataloged positions of the two infrared counterparts 2MASS J05414534-6921512 and DENIS J054145.2-692151. The 2MASS position errors are too small to be presented as an error ellipse and its position is thus represented by a cross. It can
Table 4

NIR Magnitudes and Colors of the 2MASS and DENIS Counterparts

\begin{tabular}{lccc}
\hline \hline 2MASS Counterpart & $J$ & $H$ & $K_{\mathrm{S}}$ \\
& $J-H$ & $H-K_{\mathrm{S}}$ & $J-K_{\mathrm{S}}$ \\
\hline 2MASS J05414534-6921512 & $13.31 \pm 0.04$ & $12.68 \pm 0.05$ & $12.47 \pm 0.04$ \\
& $0.63 \pm 0.06$ & $0.21 \pm 0.06$ & $0.84 \pm 0.06$ \\
\hline DENIS Counterpart & $I$ & $J$ & $K_{\mathrm{S}}$ \\
& $I-J$ & $J-K_{\mathrm{S}}$ & $I-K_{\mathrm{S}}$ \\
\hline DENIS J054145.2-692151 (a) & $14.38 \pm 0.03$ & $13.19 \pm 0.08$ & $12.41 \pm 0.14$ \\
& $1.20 \pm 0.09$ & $0.78 \pm 0.16$ & $1.98 \pm 0.14$ \\
DENIS J054145.2-692151 (b) & $14.34 \pm 0.03$ & $13.20 \pm 0.07$ & $12.16 \pm 0.11$ \\
& $1.14 \pm 0.08$ & $1.04 \pm 0.13$ & $2.18 \pm 0.11$ \\
\hline
\end{tabular}

be seen from Figure 1 that all X-ray detections are consistent in position with the uncataloged ESO-WFI source that has IR counterparts from both the 2MASS and DENIS catalogs, with only slight deviation for the flare state detection position at $\sim 2^{\prime \prime}$ from the IR source positions. Offsets of similar extent were found with the other X-ray bright source CAL 69 in the same $X M M-N e w t o n$ field, suggesting that the flare state position is compatible with the NIR counterparts identified.

In order to identify the nature of the source LMC 335, we studied the magnitudes and colors of the IR counterparts. The results are listed in Table 4. From its colors and magnitudes, it is possible to estimate the spectral type of LMC 335. Comparing our results with the 2MASS photometry of a filtered set of 461 K5 to M9.5 Galactic stars (Gelino et al. 2009), we found 90 K7 to M5.5 stars having 2MASS color indices lying within the error range reported in Table 4. The sources are mainly in the early-M types, and has a median type of M2.5, with 95.6\% (86/90) lying between K7 and M4. This is consistent with the Galactic K to M dwarf identification we derived from the stellar population study along the direction to the LMC by Nikolaev \& Weinberg (2000) using the 2MASS $J-K_{S}$ and $K_{S}$ color-magnitude diagram. Using the spectroscopic parallax relation based on a sample of MLT dwarfs by Hawley et al. (2002), and assuming a spectral type of K7 to M4, the absolute $M_{J}$ magnitude of the 2MASS counterpart is estimated to be 6.20-8.34, which transforms into a distance of 100-264 pc, with the median distance of $174 \mathrm{pc}$. Such estimation suggests that the source is not in the LMC but is in the foreground. This distance range will be adopted as the distance to the object in the subsequent analyses of X-ray/UV emission. 

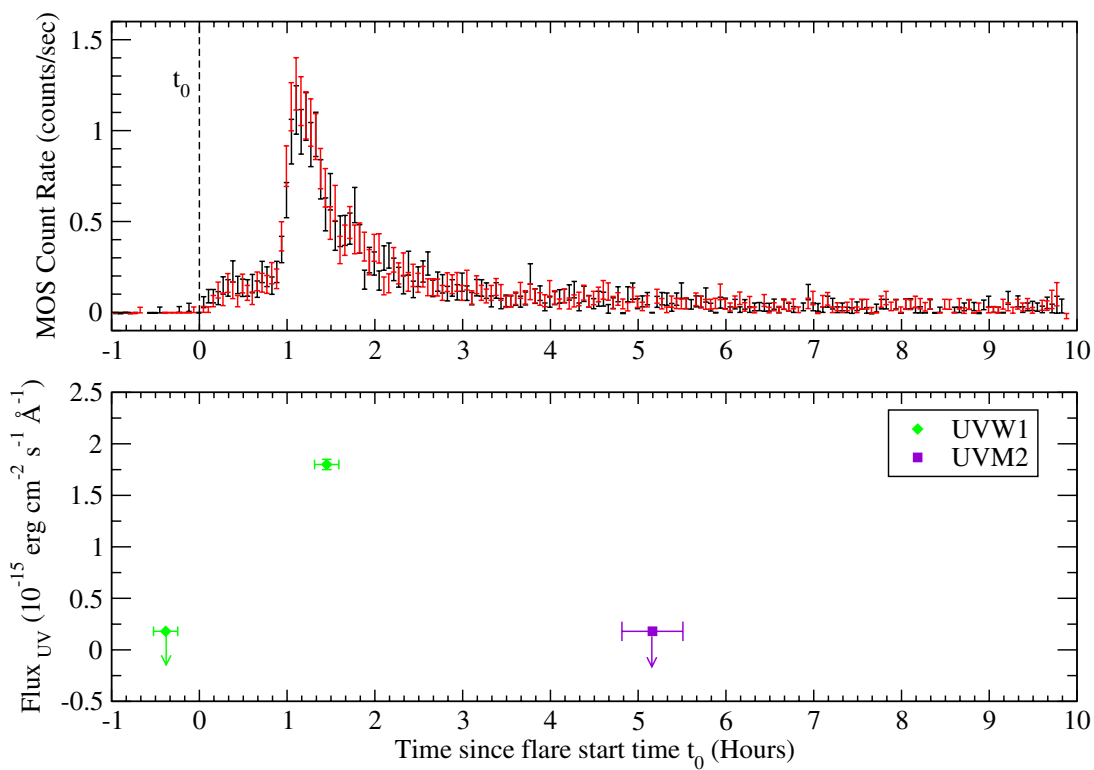

Figure 2. Upper panel: XMM-Newton EPIC MOS background-subtracted light curves (black: MOS1, red: MOS2) of LMC 335 in the $0.1-7.0 \mathrm{keV}$ band throughout the flare event. Time $t=0$ corresponds to 2000 May 27 UT 02:50; the flare start time is denoted by $t_{0}$. Time bins used are $200 \mathrm{~s}$. Lower panel: The XMM-Newton OM-derived UV fluxes/upper limits in the corresponding filters during the X-ray observations.

(A color version of this figure is available in the online journal.)

Table 5

Spectral-fitting Results of LMC 335's Flare with the XMM-Newton Detections

\begin{tabular}{|c|c|c|c|c|c|c|c|}
\hline Stage & $\begin{array}{l}\text { Duration } t_{0}+ \\
\quad(\mathrm{hr})\end{array}$ & $\begin{array}{c}n_{\mathrm{H}} \\
\left(10^{20} \mathrm{~cm}^{-1}\right)\end{array}$ & $\begin{array}{c}k T \\
(\mathrm{keV})\end{array}$ & $\chi_{v}^{2}(\mathrm{dof})$ & $\begin{array}{c}\mathrm{EM}^{\mathrm{c}} \\
\left(10^{52} \mathrm{~cm}^{-3}\right)\end{array}$ & $\begin{array}{c}\text { Flux }^{\mathrm{d}} \\
\left(10^{-13} \mathrm{erg} \mathrm{cm}^{-2} \mathrm{~s}^{-1}\right)\end{array}$ & $\begin{array}{l}\text { Luminosity }{ }^{\mathrm{c}, \mathrm{d}} \\
\left(10^{29} \mathrm{erg} \mathrm{s}^{-1}\right)\end{array}$ \\
\hline \multicolumn{8}{|c|}{ 1T APEC model results } \\
\hline $\mathrm{Q}$ & $\ldots{ }^{a}$ & $0.67^{\mathrm{b}}$ & $1.22_{-0.23}^{+0.11}$ & $1.36(22)$ & $(0.1-0.7)$ & $(0.21 \pm 0.03)$ & $(0.2-1.7)$ \\
\hline $\mathrm{P}$ & $0.00-0.90$ & $0.67^{\mathrm{b}}$ & $4.33_{-1.13}^{+2.27}$ & $1.61(10)$ & $(6.7-46.5)$ & $(11.2 \pm 1.8)$ & $(13.3-92.9)$ \\
\hline $\mathrm{R}$ & $0.90-1.13$ & $2.09_{-1.45}^{+2.13}$ & $4.88_{-1.14}^{+1.88}$ & $0.99(17)$ & $(47.4-330)$ & $(82.0 \pm 9.8)$ & $(97.7-681)$ \\
\hline D1 & $1.13-1.59$ & $0.71_{-0.60}^{+0.79}$ & $3.95_{-0.55}^{+0.62}$ & $1.44(37)$ & $(42.4-295)$ & $(70.0 \pm 5.5)$ & $(83.4-581)$ \\
\hline D2 & $1.59-10.10$ & $0.57_{-0.40}^{+0.50}$ & $3.29_{-0.27}^{+0.30}$ & $1.94(60)$ & $(4.1-28.8)$ & $(6.6 \pm 0.4)$ & $(7.8-54.6)$ \\
\hline $\mathrm{F}$ & $0.96-1.52$ & $1.23_{-0.60}^{+0.75}$ & $4.29_{-0.48}^{+0.71}$ & $1.19(49)$ & $(49.5-345)$ & $(83.4 \pm 5.6)$ & $(99.4-693)$ \\
\hline \multicolumn{8}{|c|}{ 2T APEC model results } \\
\hline \multirow[t]{2}{*}{$\bar{P}$} & $0.00-0.90$ & $0.67^{\mathrm{b}}$ & $0.28_{-0.28}^{+1.20}$ & $1.45(8)$ & $(0.4-2.8)$ & $(11.7 \pm 2.1)$ & $(14.0-97.4)$ \\
\hline & & & $5.21_{-1.82}^{+2.47}$ & & $(6.1-42.6)$ & & \\
\hline \multirow[t]{2}{*}{$\mathrm{R}$} & $0.90-1.13$ & $1.91_{-1.35}^{+1.92}$ & $1.01_{-0.69}^{+3.32}$ & $0.98(15)$ & $(1.5-10.2)$ & $(83.2 \pm 10.1)$ & $(99.5-693)$ \\
\hline & & & $5.83_{-1.25}^{+2.31}$ & & $(44.2-308)$ & & \\
\hline \multirow[t]{2}{*}{ D1 } & $1.13-1.59$ & $0.67_{-0.63}^{+0.88}$ & $0.95_{-0.17}^{+0.13}$ & $0.93(35)$ & $(2.6-18.2)$ & $(70.8 \pm 5.8)$ & $(84.7-591)$ \\
\hline & & & $4.66_{-0.80}^{+1.39}$ & & $(36.9-257)$ & & \\
\hline \multirow[t]{2}{*}{ D2 } & $1.59-10.10$ & $0.67_{-0.45}^{+0.57}$ & $0.76_{-0.16}^{+0.10}$ & $1.09(58)$ & $(0.3-1.8)$ & $(6.7 \pm 0.40)$ & $(8.0-55.6)$ \\
\hline & & & $3.61_{-0.36}^{+0.49}$ & & $(3.6-25.1)$ & & \\
\hline \multirow[t]{2}{*}{$\mathrm{F}$} & $0.96-1.52$ & $1.14_{-0.63}^{+0.80}$ & $1.06_{-0.22}^{+0.28}$ & $0.99(47)$ & $(2.3-16.1)$ & $(84.2 \pm 5.9)$ & $(101-702)$ \\
\hline & & & $5.07_{-0.86}^{+1.61}$ & & $(44.8-312)$ & & \\
\hline
\end{tabular}

Notes. The error ranges of all parameters give the $90 \%$ confidence intervals.

${ }^{\text {a }}$ The spectral properties are derived from previous non-flare PN observations, as listed in Table 1.

$\mathrm{b}$ The value was fixed to the average of the decay stages in the $2 \mathrm{~T}$ model.

${ }^{c}$ Parameters are shown in ranges corresponding to source distance of 100-264 pc.

${ }^{\mathrm{d}} \mathrm{X}$-ray fluxes and luminosities are for the $0.1-7.0 \mathrm{keV}$ band.

\subsection{Timing Analysis}

The X-ray flare event of LMC 335 on 2000 May 27 was captured by the XMM-Newton MOS cameras in its entirety. The $0.1-7.0 \mathrm{keV}$ background-subtracted X-ray light curve with $200 \mathrm{~s}$ binning is presented in the upper panel of Figure 2 (black:
MOS1; red: MOS2). The time axis starts at 2000 May 27 UT 02:50 when the precursor phase $(\mathrm{P})$ started, this will be referred to as the flare start time $t_{0}$ from hereafter. The quiescent state $(\mathrm{Q})$ of the source persisted during the first hour of observations with flux beneath our detection limit. Only a $3 \sigma$ upper limit count rate of about $6 \times 10^{-3} \mathrm{~s}^{-1}$ could be obtained. This is consistent with 
the expected average quiescent count rate computed from all other XMM-Newton MOS detections at $(4.0 \pm 0.7) \times 10^{-3} \mathrm{~s}^{-1}$. The Chandra and Swift quiescent observations also produced consistent values, with PIMMS-converted (v4.4) XMM-Newton MOS count rates being $(5.7 \pm 0.9) \times 10^{-3}$ and $(3.9 \pm 0.6)$ $\times 10^{-3}$, respectively, assuming a $1.1 \mathrm{keV}(\sim 12 \mathrm{MK})$ APEC plasma model (cf. Table 5, Section 3.3). At $\sim 1 \mathrm{hr}$ after the start of the observation, the X-ray count rate suddenly rose to a precursor level (P) of $0.1-0.2 \mathrm{~s}^{-1}$. At $\sim 55$ minutes after $t_{0}$, the flare entered the rise $(\mathrm{R})$ phase when the count rate rose sharply during $\sim 800 \mathrm{~s}(\sim 13$ minutes $)$ from the precursor level to over $1.1 \mathrm{~s}^{-1}$ at the flare peak. The flare flux then entered a decay phase starting $1.13 \mathrm{hr}$ after $t_{0}$ and continuing until the end of the observation almost $10 \mathrm{hr}$ later.

The OM provides simultaneous UV observations during the $\mathrm{X}$-ray flare of LMC 335. Flux variation in the UV band during the flare is shown in Figure 2 (lower panel). Detailed detected count rate, magnitude, and flux, as well as the $3 \sigma$ upper limits, are listed in Table 2. Only upper limits could be derived for the $U V W 1$ observation taken during the quiescent phase of the flare, and the $U V M 2$ data taken $\sim 5 \mathrm{hr}$ after $t_{0}$. The only detection was the $U V W 1$ image taken $\sim 1.5 \mathrm{hr}$ after $t_{0}$ during the decay 1 (D1) phase, when a UV flux rose by at least one order of magnitude from the quiescent phase upper limit. Mitra-Kraev et al. (2005) studied the relation between X-ray and UV energy budget and found that the luminosity increases in X-ray and UV (measured in $U V W 1$ band) during a stellar flare can be expressed as $\Delta L_{\mathrm{X}} \propto \Delta L_{\mathrm{UV}}^{\alpha}$, where $\alpha \sim 1.1-1.2$. While the time resolution of our UV data is not high enough for more detailed analysis, the observed flux increases in X-ray and UV are consistent with the power-law relation.

To facilitate a quantitative study of the behavior of the flare during the decay phase, the light-curve data points taken after $t_{0}+1.6 \mathrm{hr}$ were rebinned into intervals of $1000 \mathrm{~s}$ to give better statistics. The MOS1-MOS2 averaged count rates from the peak of emission $1.13 \mathrm{hr}$ after $t_{0}$ are fitted with various exponential decay functions. We found that the data could be fit well with a two-component exponential decay function (reduced $\chi^{2}$, dof $=$ $1.01,35)$ while the single-component model can be ruled out due to apparent deviation especially after the first hour of decay (reduced $\chi^{2}$, dof $=17.07,37$ ). Initially the drop was rapid with an $e$-folding time (and $1 \sigma$ error) of $28.6_{-1.3}^{+1.9}$ minutes, the decay then slowed down to an $e$-folding time of $4.3_{-0.6}^{+3.5} \mathrm{hr}$. The $\mathrm{X}$-ray light curve of the flare and the exponential fit are shown in Figure 3.

Based on the exponential fits to the X-ray light curve, we further divided the decay phase into the decay 1 (D1) phase from the flare peak to include the first $e$-folding time of the fast decay component, followed by the decay 2 (D2) phase until the end of the observations. The time boundaries of the quiescence $(\mathrm{Q})$, precursor $(\mathrm{P})$, rise (R), decay 1 (D1), and decay (D2) phases are marked by black vertical dashed lines in Figure 3. To have a more detailed analysis of the conditions of the flare during its peak, an additional flare (F) phase overlapping with $\mathrm{R}$ and D1, starting from 10.0 minutes before the peak to 23.4 minutes after flare peak, is defined as the interval during which the detected $\mathrm{X}$-ray count rate is higher than half of the peak value (marked with dotted lines in Figure 3). The inset in Figure 3 shows a zoomed-in view of the light curve near the flare peak. At the beginning of phase D1, a slight excess in counts is observed at about $1.3 \mathrm{hr}$ after $t_{0}$, which could possibly come from another very brief rise phase after the main peak. Such multiple peak light curves had been detected in other stellar flares with

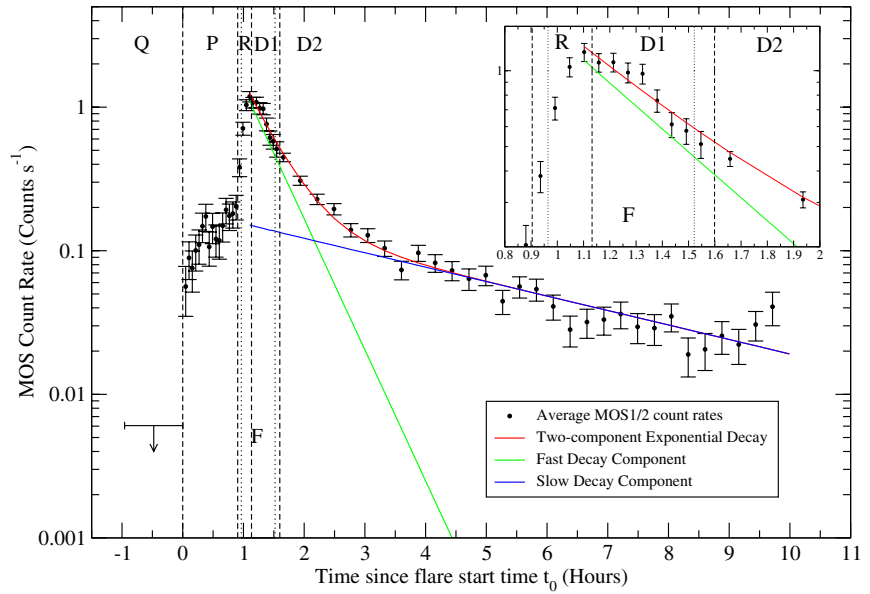

Figure 3. MOS1 and MOS2 averaged light curve of LMC 335 is replotted in logarithmic scale overlaid with the exponential fits. Time $t=0$ refers to 2000 May 27 UT 02:50, the flare start time $t_{0}$ as denoted in Figure 2. The observation is partitioned into five phases: quiescence $(\mathrm{Q})$, precursor $(\mathrm{P})$, rise $(\mathrm{R})$, and decay 1 and $2(\mathrm{D} 1-\mathrm{D} 2)$, marked by black dashed lines. The additional flare (F) phase bounded by dotted lines is added to study the properties of LMC 335 during the peak state. The time bins for D2 have been widened from $200 \mathrm{~s}$ to $1000 \mathrm{~s}$ for better statistics and visualization. Detailed evaluation of the light curve near the flare peak is shown in the inset figure.

(A color version of this figure is available in the online journal.)

comparable rise times (Pandey \& Singh 2008; Welsh et al. 2007). On the other hand, this slight excess could certainly also have been resulted from the statistical fluctuations of the data.

This flare observation of LMC 335 was fortuitously captured near the beginning of a long XMM-Newton exposure, thus allowing continuous observations of the first $10 \mathrm{hr}$ of the flare event. From Figure 3, it is obvious that the X-ray emission still had not returned to the quiescent emission level by the end of the observation. The average MOS count rate for the last hour before the end of observations is $0.029 \pm 0.006 \mathrm{~s}^{-1}$, which is a factor of five to seven higher than the expected quiescent rate of $0.004 \mathrm{~s}^{-1}$. This could originate from low-level flares persistent in the active regions after the main event.

\subsection{X-Ray Spectral Analysis}

With the count rate at a low level for most of the duration of the flare, hardness ratios (HRs) were first computed to study the overall X-ray energy distribution of the event and to provide model-independent information for comparisons with other flares and other X-ray sources. The flare is soft, with $\mathrm{X}$-ray photons in the energy range $0.1-2.0 \mathrm{keV}$ making up $\sim 80 \%$ of all photons collected during the observation. The HR is defined as HR $=H / S$, where $S$ and $H$ are, respectively, the $0.1-2.0 \mathrm{keV}$ and $2.0-7.0 \mathrm{keV}$ count rates averaged from the MOS1 and MOS2 observations. Since LMC 335 was below the detection limit during the first hour of the flare XMM-Newton MOS observations, the HR for the quiescent phase is averaged from the non-flare PN XMM-Newton observations for proper comparison with the spectral-fitting results. Figure 4 shows the time evolution of the HR of the flare during the five phases from pre-flare quiescence $(\mathrm{Q})$ through the decay phases, with the flare phase $(F)$ data point also shown for reference. We observed a sharp rise in HR from Q of $0.09 \pm 0.05$ to phase $\mathrm{P}$ of $0.45 \pm 0.06$, reaching a maximum at phase $\mathrm{R}$ of $0.47 \pm$ 0.05. The value of HR then dropped through the $\mathrm{F}$ and the two decay phases, settling back near the quiescent level at $0.12 \pm 0.01$ for the D2 phase. The results imply that the flare 


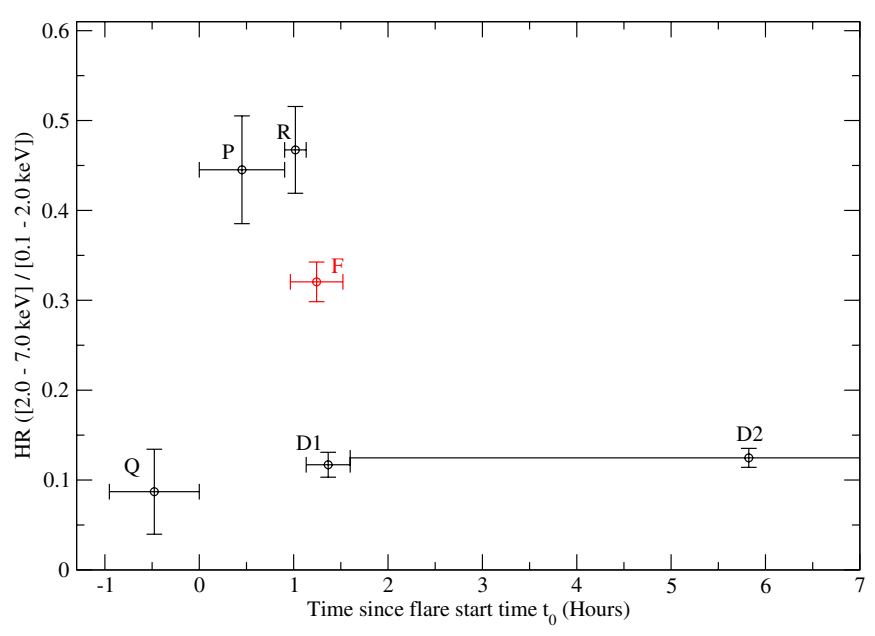

Figure 4. Evolution of X-ray hardness ratio (HR) during the six representative phases from quiescence (Q) to decay 2 (D2). The averaged HR from the non-flare $\mathrm{PN} X M M-N e w t o n$ observations is used to represent phase $\mathrm{Q}$.

(A color version of this figure is available in the online journal.)

in hard X-rays commenced simultaneously with the flare of the softer components, but decayed away much more quickly. This observed softening in X-ray after the $\mathrm{R}$ phase is common among late-type stellar flares (Reale et al. 2004; Trenholme et al. 2004; Schmitt \& Liefke 2002).

In addition to computing the HRs, we have investigated the $\mathrm{X}$-ray spectra of the flare in each phase. Identical procedures were followed for the generation of X-ray spectra in each phase except for the different numbers of minimum counts used to bin the spectra. The single-temperature (1T) and two-temperature (2T) thermal emission APEC models were used to analyze the spectra, except for the $\mathrm{Q}$ phase spectrum in which only the $1 \mathrm{~T}$ model was carried out to derive a best-fit temperature of $1.22 \mathrm{keV}(14.2 \mathrm{MK})$. The $2 \mathrm{~T}$ model was found to provide better fits than the $1 \mathrm{~T}$ model for all the other phases, with significant improvements for phases D1, D2, and F. As the fits were insensitive to the choice of metal abundance parameter, the solar abundance was therefore used. The emission measure $(\mathrm{EM})$ is derived from the normalization of the APEC model fitting in XSPEC and the assumed source distance range.

We observed that the high-temperature components of $\sim 5 \mathrm{keV}$ emerged in the $\mathrm{P}$ and $\mathrm{R}$ phases, followed by a slight decline during the decay phases to $\sim 3 \mathrm{keV}$ while the lowtemperature components stayed at $\sim 1 \mathrm{keV}$. The dominance of the hotter components is revealed from the consistently larger EM throughout the event as well as the biases in the fitted temperatures toward the hot range with the $1 \mathrm{~T}$ model. Also, we observed an increase in EM for the cooler components above the quiescent value particularly in the R, D1, and F phases, which could be associated with the flare. Noticeable increases (by factors of 4-9) in EM of the low-temperature components from quiescence to flare peaks had been observed in other flares (Robrade et al. 2010; Gupta et al. 2011), but not as high as observed for this object (factor of $\sim 23$ ). Since the spectra from MOS1 and MOS2 are very similar, only the MOS1 spectra taken during each phase are shown in Figure 5. Data from MOS1 and MOS2 were fitted together to provide better statistics and the quiescent spectrum is obtained by previous non-flare PN observations. The best-fit parameters of the two models in different flare phases are summarized in Table 5 with all errors given in $90 \%$ confidence intervals as computed in XSPEC. The spectra had also been fitted by the equilibrium plasma model
Mewe-Kaastra-Liedahl (MeKaL), resulting in similar best-fit parameters.

Due to the limited signals in the quiescent $(\mathrm{Q})$ and the precursor $(\mathrm{P})$ phases, the line-of-sight neutral hydrogen column density $\left(n_{\mathrm{H}}\right)$ could not be constrained by the fitting. Fixing $n_{\mathrm{H}}$ to values below $10^{21} \mathrm{~cm}^{-2}$ did not induce significant changes to the fitted plasma temperature, while the fits were poor (with reduced $\chi^{2} \geqslant 3$ ) for $n_{\mathrm{H}}$ values above a few times of $10^{21} \mathrm{~cm}^{-2}$. The $n_{\mathrm{H}}$ in quiescent and precursor states were thus fixed to be the average of the best-fit values from the two decay phases D1 and D2 at $0.67 \times 10^{20} \mathrm{~cm}^{-2}$ for both the $1 \mathrm{~T}$ and $2 \mathrm{~T}$ models. The value from the rise phase $(\mathrm{R})$ was not used for the estimation of $n_{\mathrm{H}}$ due to the unstable physical conditions during the period and the resulting bigger uncertainties of the fitted parameters. By using a fixed $n_{\mathrm{H}}$ in all phases, the other spectral parameters are similar to those obtained with floating $n_{\mathrm{H}}$. The best-fit values of $n_{\mathrm{H}}$ from these spectra were about one order of magnitude lower than the weighted average values of hydrogen column density along that line of sight toward the LMC estimated from the Leiden/Argentine/Bonn survey $(3.37 \times$ $10^{21} \mathrm{~cm}^{-2}$ ) (Kalberla et al. 2005) and the Dickey \& Lockman Galactic survey $\left(6.76 \times 10^{20} \mathrm{~cm}^{-2}\right)$ (Dickey \& Lockman 1990). This provides additional supporting argument that the source is indeed located in the foreground instead of within the LMC.

The luminosity of LMC 335 at the quiescent state $L_{\mathrm{X}, \mathrm{q}}=(0.2-1.7) \times 10^{29} \mathrm{erg} \mathrm{s}^{-1}(0.1-7.0 \mathrm{keV})$ measured by $X M M-N e w t o n$ is comparable with the PIMMS implied value from the ROSAT HRI detected value of $2.4 \times 10^{29} \mathrm{erg} \mathrm{s}^{-1}$ (0.1-2.4 keV) assuming the $1.1 \mathrm{keV}$ APEC model as deduced for quiescence. Chandra and Swift observations also support the relatively stable X-ray activity of the source in quiescence, with inferred luminosities of $1.8 \times 10^{29} \mathrm{erg} \mathrm{s}^{-1}(0.08-10.0 \mathrm{keV})$ and $1.3 \times 10^{29} \mathrm{erg} \mathrm{s}^{-1}(0.2-10.0 \mathrm{keV})$, respectively, assuming the same spectral model.

Combining and averaging from the two MOS observations for the flare during the entire duration of observation, the flux of the source is $(8.8 \pm 0.4) \times 10^{-13} \mathrm{erg} \mathrm{s}^{-1} \mathrm{~cm}^{-2}$, implying a total emitted X-ray energy of $(0.4-2.9) \times 10^{35}$ erg assuming a distance to the source of (100-264) pc and the total exposure time of $40 \mathrm{ks}$.

To characterize the X-ray activity, the $\log \left(L_{\mathrm{X}} / L_{\text {bol }}\right)$ ratios at different stages were computed and compared. To estimate the bolometric luminosities from the bolometric correction (BC) factor, the $\mathrm{BC}_{K}-\left(J-K_{S}\right)$ relation derived from $51 \mathrm{M} 0-\mathrm{L} 7$ stars with $0.75 \leqslant\left(J-K_{S}\right) \leqslant 1.60$ by Leggett et al. (2001) was used. To construct the BC relation, they computed the bolometric luminosities of the sample stars by integrating the observed IR spectra, with the short-wavelength ends linearly extrapolated to zero flux at zero wavelength and the long-wavelength ends approximated by the Rayleigh-Jeans tail. Using the IR colors of the 2MASS counterpart in Table 4, the $\mathrm{BC}_{K}$ was computed to be 2.63 , and this transforms into an apparent bolometric magnitude of 15.10. Combining with the source distance of (100-264) pc, an absolute bolometric magnitude $M_{\text {bol }}$ of (8.0-10.1) could be determined, implying the value of $\log \left(L_{\mathrm{bol}} / L_{\odot}\right)$ from -2.1 to -1.3 . The range agrees closely with the estimates of -1.27 and -1.99 for M0/M1 and M3/M4 stars, respectively, in the GALEX M dwarf UV flare survey (Welsh et al. 2007). The APEC model results were used for the estimation of the total $\mathrm{X}$-ray luminosity $L_{\mathrm{X}}$ to yield $\log \left(L_{\mathrm{X}, \mathrm{q}} / L_{\mathrm{bol}}\right)=-3.1$ during quiescent state and $\log \left(L_{\mathrm{X} \text {,flare }} / L_{\mathrm{bol}}\right)=-0.4$ in the flare state, suggesting a significant rise in X-ray activity. 


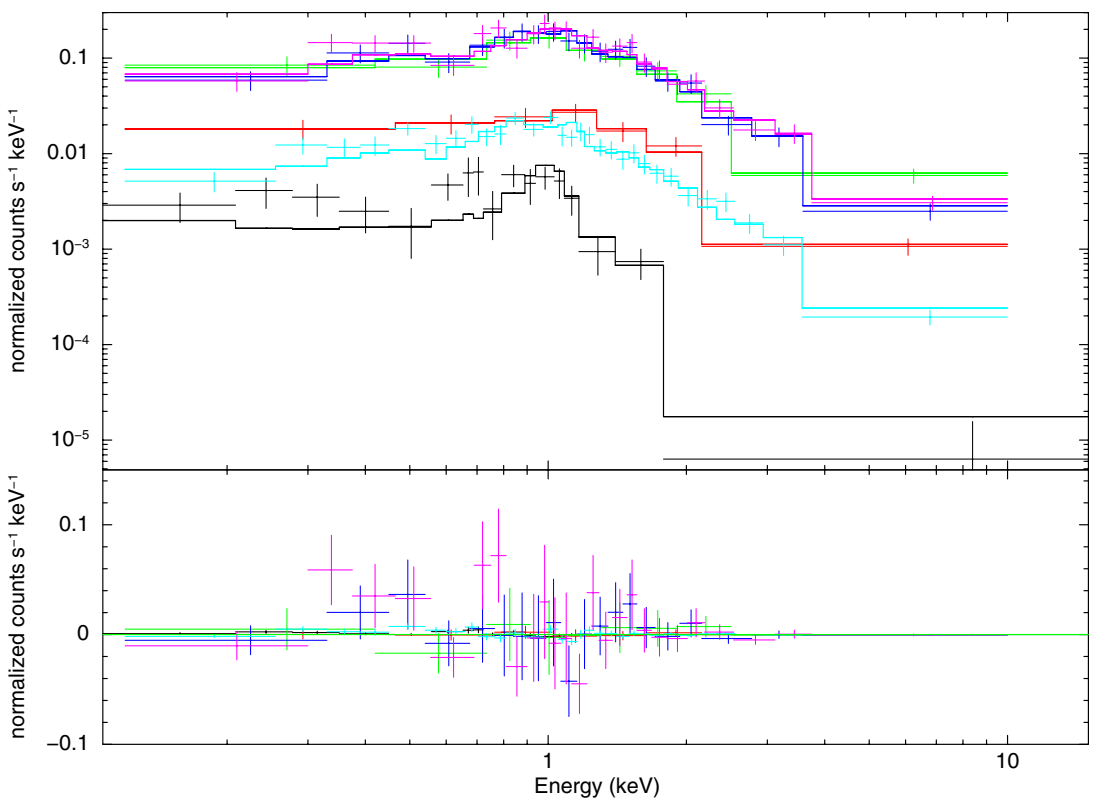

Figure 5. XMM-Newton MOS1 background-subtracted spectra with best-fit $1 \mathrm{~T}$ APEC model for quiescence (black); and 2T APEC model for precursor (red), rise (green), decay 1 (blue), decay 2 (cyan), and flare (magenta) phases.

(A color version of this figure is available in the online journal.)

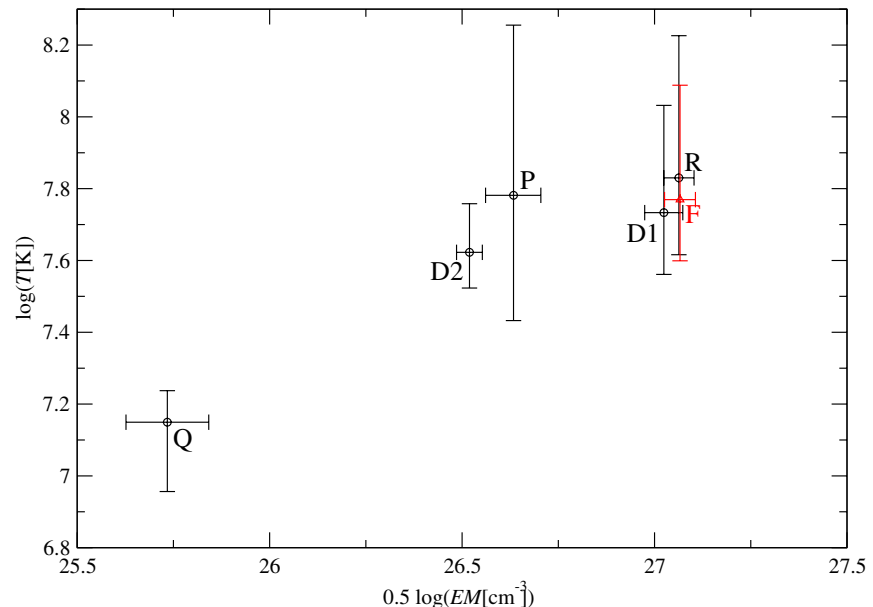

Figure 6. Time evolution of temperature and the emission measure (EM) during the six representative phases from quiescence (Q) to decay 2 (D2) as derived for a reference spectral type of $\mathrm{M} 2.5(174 \mathrm{pc})$. The data point for quiescent phase is derived from the 1T APEC model; parameters for all other phases are obtained from the high-temperature components of the $2 \mathrm{~T}$ APEC fits. The error bars for the EM values include only the uncertainties in XSPEC normalization, the uncertainty in source distance is not included. For spectral types of K7 (264 pc) and M4 $(100 \mathrm{pc})$, the values on the EM axis are offset by 0.18 and -0.24 , respectively, with the relative positions of data points unchanged.

(A color version of this figure is available in the online journal.)

The evolution of the flare from quiescent to the decay phase is traced on the $\log T-\log \sqrt{\mathrm{EM}}$ plane as shown in Figure 6. All the EM values were taken from the dominant high-temperature components of the $2 \mathrm{~T}$ spectral fits except for phase Q. Data points on Figure 6 are calculated based on the reference spectral type of M2.5 (174 pc), and the EM error bars include only spectral-fitting errors with XSPEC, while errors due to the uncertain source distance are not included. It is found that both the plasma temperature and EM increased significantly from Q to $P$ phase. During the flare phases from $\mathrm{P}$ to $\mathrm{D} 2$, the temperature stayed roughly constant at $\sim(5-6) \times 10^{7} \mathrm{~K}$ while the EM fluctuated by about an order of magnitude around $10^{54} \mathrm{~cm}^{-3}$. Similar evolution trends in both EM and temperature were also observed in the X-ray flare of the brown dwarf LP 412-31 (Stelzer et al. 2006) and the X-ray/optical flare of the lateM dwarf Proxima Centauri (Reale et al. 2004). The relatively large errors of the spectral fitting prohibit more detailed studies of the evolution structure in the $\log T-\log \sqrt{\mathrm{EM}}$ plane, but the general trend is clear. For source distances of 264 pc (K7) and $100 \mathrm{pc}(\mathrm{M} 4)$, the relative positions between data points remain unchanged while the values on the EM axis offset by 0.18 and -0.24 , respectively.

As the current signal to noise is not sufficient for detailed modeling, we studied the physical properties of the flare based on the Haisch's simplified approach (HSA; Haisch 1983; Mullan et al. 2006). In this simplified parameterized scheme, the length of the flare loop can be estimated based only on the EM and the flare decay timescale $\tau_{\mathrm{d}}$. The loop lengths derived here represent the upper limits since multiple loops could be present while the numbers presented are for the single-loop scenario; together with the fact that the determined EM in the flare phase (F) should also include contributions, albeit small, from the precursor phase (P). In addition to the work by Mullan et al. (2006) with EUVE, Pandey \& Singh (2012) adopted the HSA to study a number of $\mathrm{X}$-ray flares from CVn-type binaries captured by XMM-Newton, while Schmitt et al. (1987) also tested the reliability of HSA with solar X-ray flares using EM values measured from Einstein. Using the D1 phase decay timescale derived from the MOS light curve and the sum of $\mathrm{F}$ phase EM, the flare loop length during the $\mathrm{F}$ phase is estimated to be $(3.4-5.6) \times 10^{10} \mathrm{~cm}$. Taking the stellar radii of M4 and $\mathrm{K} 7$ stars to be $0.4 R_{\odot}$ and $0.7 R_{\odot}$ (Lacy 1977; White et al. 1989; Ségransan et al. 2003), the size of the loop is estimated to be 1.1-1.3 times of the stellar radius. The range of the flare loop length in stellar radius is computed by the loop length and the stellar radius estimated for the associated spectral type. This is compatible with the picture that long-loop flares on main-sequence stars occur mainly on $\mathrm{M}$ dwarfs as proposed by Mullan et al. (2006). 


\section{DISCUSSION AND CONCLUSIONS}

In this paper, we report the discovery and the multiwavelength investigation of an X-ray/UV stellar flare from the K7 to M4 dwarf star LMC 335. The long, continuous X-ray observation of the source from the onset of the flare makes this event unique among those presented in the existing literature. While concurrent flare observations in X-ray and optical/UV bands had also been reported on later type M8 and M8.5 stars (Stelzer et al. 2006; Robrade et al. 2010), the current study is one of the best observed samples of late-K and early-M dwarfs with a full $11 \mathrm{hr} \mathrm{X}$-ray coverage from quiescence to late decay phase together with a well-observed precursor.

Quiescent emission from the source is found from multiple observations in X-ray, optical, and IR, with a counterpart identified in both the 2MASS and DENIS catalogs. The IR photometry and colors characterize the source as a K7 to M4 dwarf star, supporting the conclusion that the observed high-energy flare originated from the stellar coronae. In quiescence, LMC 335 has an X-ray luminosity of $L_{\mathrm{X}, \mathrm{q}} \approx(0.2-1.7) \times 10^{29} \mathrm{erg} \mathrm{s}^{-1}$ and a temperature of $\sim 12 \mathrm{MK}$ from the XMM-Newton data. The values are typical for M dwarfs (Pallavicini et al. 1990; Schmitt et al. 1995; Schmitt \& Liefke 2004), and are consistent with those deduced from both Chandra and Swift observations in quiescence. The X-ray luminosity in quiescence and the bolometric luminosity of LMC 335 are found to be closely consistent with the K7-M4 subsample studied by Pallavicini et al. (1990) and Tsikoudi et al. (2000). Moreover, the observed $\log \left(L_{\mathrm{q}} / L_{\text {bol }}\right)$ agree almost perfectly with the tight correlation of the two quantities deduced by Pallavicini et al. (1990) for late-K to M dwarfs, thus further confirming the K7-M4 classification of LMC 335. The distance of LMC 335 based on spectroscopic parallax and the $n_{\mathrm{H}}$ limit was estimated to be 100-264 pc. Consistent results obtained in the timing and spectral analysis with similar dwarf flares further strengthen the conclusion that LMC 335 is indeed in the foreground instead of residing in the LMC.

Clear precursor emission before the commencement of the main flare event was observed, with released X-ray energy of $\sim(0.5-3.2) \times 10^{34} \mathrm{erg}$, or about one-tenth of the total. Similar precursor phases have also been observed from the ultracool dwarf LP 412-31 (Stelzer et al. 2006) and from the Sun (Joshi et al. 2011), suggesting an intimate spatial relation between the pre-flare magnetically active regions and the main flare. The X-ray luminosity then sharply increased through the $\mathrm{R}$ phase to reach values of $(1.0-7.0) \times 10^{31} \mathrm{erg} \mathrm{s}^{-1}$ and $(0.3-1.7) \times 10^{31} \mathrm{erg} \mathrm{s}^{-1}$ in X-ray and UV, respectively. During the flare peak, the X-ray luminosity of LMC 335 was $\sim 400$ times higher than the quiescent value. The strongest increase recorded by Pallavicini et al. (1990) was only $\sim 20$, while more recently observed X-ray flares exhibited flux increases by factors ranging from few (Pandey \& Singh 2008) to tens (Schmitt \& Liefke 2002; Güdel et al. 2004; Stelzer et al. 2006; Wargelin et al. 2008; Robrade et al. 2010; Gupta et al. 2011), and up to hundreds (Favata et al. 2000; Fleming et al. 2000; Rutledge et al. 2000) to $\sim 7000$ (Osten et al. 2010). Furthermore, the quiescent luminosities of these sources $\left(10^{25}-10^{28} \mathrm{erg} \mathrm{s}^{-1}\right)$ place LMC 335 at the energetic end of the X-ray flare samples. The loop length of LMC 335 is estimated from the HSA scheme to be comparable or bigger than the stellar radius, classifying our current flare under the "long-loop" category comparable to the $\sim 1-3$ times stellar radius reported for less luminous flares (Schmitt \& Liefke 2002; Mullan et al. 2006; Wargelin et al. 2008).
The long-time continuous X-ray coverage of the flare event allows for detailed light-curve analysis. After the main flare peak, there could be a brief rise phase, arising possibly from the superposition of a less intense secondary flare on the main one. This could imply a more complicated spatial structure of the corona than the compact single-loop flaring scenario. More prominent multi-peak X-ray/UV flares had been observed for a number of $G$ to $K$ dwarfs (Pandey \& Singh 2008) and $M$ dwarfs (Welsh et al. 2007). For LMC 335, the drop in flux after the flare peak could be best characterized by a two-component exponential decay with $e$-folding timescales of $\sim 28$ minutes and $\sim 4 \mathrm{hr}$, and the single-component decay model is ruled out. Similar two-stage flare decays were observed in some $\mathrm{M}$ dwarf X-ray flares including the M6 Proxima Centauri (Reale et al. 2004), M3.5 Ross 154 (Wargelin et al. 2008), and the M8 LP 412-31 (Stelzer et al. 2006). There are different interpretations of the change in decay timescale. Observations of large solar flares suggested that the slower decay may have resulted from the reconnected loops of the main flare, which evolve differently (Aschwanden \& Alexander 2001), or the overlapping with another ongoing flare event (Reale et al. 2004). On the other hand, there are flares in which single-component decay models provide satisfactory fits. Their decay timescales range from $\sim 0.1 \mathrm{ks}$ to $\gtrsim 10 \mathrm{ks}$. Finally, the observed count rate still exceeded the quiescent level by a factor of $\sim 5-7$ at the end of observation after $9 \mathrm{hr}$ of decay. Such late-time decay emission could be coming from continuous low-level flare events occurring in the active regions left behind by the main flare.

The evolution of $n_{\mathrm{H}}$ at different phases cannot be constrained with the current data set. Spectral fitting with 2T APEC model supports the parameterization of the flare spectra into a lowtemperature component and a time-dependent high-temperature flare component with similar spectral shapes and comparable parameters to other late-M objects (Hambaryan et al. 2004; Stelzer et al. 2006; Robrade \& Schmitt 2009; Robrade et al. 2010; Gupta et al. 2011). The cooler components ( $10 \mathrm{MK})$ are observed to have enhanced EM, along with the emergence of the hot components ( $60 \mathrm{MK})$ with EM of up to (0.4-3.1) $\times$ $10^{54} \mathrm{~cm}^{-3}$ during flare peak. The total X-ray energy released is $\sim(0.4-2.9) \times 10^{35} \mathrm{erg}$. While only very few K7-M4 dwarf $\mathrm{X}$-ray flares have been reported, the observed properties of LMC 335 place it at the energetic end of the limited sample.

The simultaneous UV observations show significant brightening in the UV band. Focusing primarily on the temporal coincidence between light curves in X-ray and UV, Mitra-Kraev et al. (2005) found an approximate time lag of $\sim 10$ minutes in $\mathrm{X}$-ray. The low sampling rate of our UV data for LMC 335 could not confirm such possible lag. On the other hand, the lower limit of the X-ray to UV energy loss ratio derived for the quiescence phase and the ratio observed during the flare phase is consistent with the description of the quiescent state as the superposition of continuous low-energy small-scale flares known as micro-flares (Parker 1988) or nano-flares depending on the timescales.

We thank the anonymous referee for the helpful comments on the characterization of the spectral type of the source. B.T.H.T. acknowledges valuable discussions with Dr. Jifeng Liu and Dr. Frank Primini, and the support from HKU under the grant of Postgraduate Studentship. A.K.H.K. gratefully acknowledges support from the National Science Council of the Republic of China (Taiwan) through grant NSC100-2628-M-007-002-MY3 and a Kenda Foundation Golden Jade Fellowship. This research has made use of data and software provided by the High Energy 
Astrophysics Science Archive Research Center (HEASARC), which is a service of the Astrophysics Science Division at NASA/GSFC and the High Energy Astrophysics Division of the Smithsonian Astrophysical Observatory. This research has also made use of the NASA/IPAC Infrared Science Archive, which is operated by the Jet Propulsion Laboratory, California Institute of Technology, under contract with the National Aeronautics and Space Administration. This was also supported by the use of observations made with the European Southern Observatory telescopes obtained from the ESO/ST-ECF Science Archive Facility. This investigation was supported by the RGC/GRF grant 704709 from the government of the Hong Kong SAR.

\section{REFERENCES}

Allred, J. C., Hawley, S. L., Abbett, W. P., \& Carlsson, M. 2006, ApJ, 644, 484 Aschwanden, M. J., \& Alexander, D. 2001, Sol. Phys., 204, 91

Audard, M., Güdel, M., Sres, A., Raassen, A. J. J., \& Mewe, R. 2003, A\&A, 398, 1137

Benz, A. O., \& Güdel, M. 2010, ARA\&A, 48, 241

Berger, E., Basri, G., Fleming, T. A., et al. 2010, ApJ, 709, 332

Cabrera-Lavers, A., \& Garzón, F. 2003, A\&A, 403, 383

DENIS Consortium 2005, VizieR Online Data Catalog, 2263, 0

Dickey, J. M., \& Lockman, F. J. 1990, ARA\&A, 28, 215

Favata, F., Micela, G., \& Reale, F. 2001, A\&A, 375, 485

Favata, F., Reale, F., Micela, G., et al. 2000, A\&A, 353, 987

Fleming, T. A., Giampapa, M. S., \& Schmitt, J. H. M. M. 2000, ApJ, 533, 372

Gelino, C. R., Kirkpatrick, J. D., \& Burgasser, A. J. 2009, in AIP Conf. Ser. 1094, Cool Stars, Stellar Systems and the Sun, ed. E. Stempels (Melville, NY: AIP), 924

Guainazzi, M. 2011, XMM-Newton Calibration Technical Note (Tech. Rep. XMM-SOC-CAL-TN-0018; Villafranca del Castillo, Spain: ESA-ESAC)

Güdel, M., Audard, M., Reale, F., Skinner, S. L., \& Linsky, J. L. 2004, A\&A, 416,713

Gupta, A., Galeazzi, M., \& Williams, B. 2011, ApJ, 731, 63

Haisch, B. M. 1983, in IAU Colloq. 71, Activity in Red Dwarf Stars, ed. P. B. Byrne \& M. Rodono (Dordrecht: Reidel), 255

Hambaryan, V., Staude, A., Schwope, A. D., et al. 2004, A\&A, 415, 265

Hawley, S. L., Covey, K. R., Knapp, G. R., et al. 2002, AJ, 123, 3409

Huenemoerder, D. P., Canizares, C. R., \& Schulz, N. S. 2001, ApJ, 559, 1135
Joshi, B., Veronig, A. M., Lee, J., et al. 2011, ApJ, 743, 195

Kahn, S. M., Mason, K. O., Bowyer, C. S., et al. 1979, ApJ, 234, 107

Kalberla, P. M. W., Burton, W. B., Hartmann, D., et al. 2005, A\&A, 440, 775

Lacy, C. H. 1977, ApJS, 34, 479

Leggett, S. K., Allard, F., Geballe, T. R., Hauschildt, P. H., \& Schweitzer, A. 2001, ApJ, 548, 908

Mitra-Kraev, U., Harra, L. K., Güdel, M., et al. 2005, A\&A, 431, 679

Mullan, D. J., Mathioudakis, M., Bloomfield, D. S., \& Christian, D. J. 2006, ApJS, 164, 173

Nikolaev, S., \& Weinberg, M. D. 2000, ApJ, 542, 804

Osten, R. A., Godet, O., Drake, S., et al. 2010, ApJ, 721, 785

Osten, R. A., Hawley, S. L., Allred, J., et al. 2006, ApJ, 647, 1349

Pallavicini, R., Tagliaferri, G., \& Stella, L. 1990, A\&A, 228, 403

Pandey, J. C., \& Singh, K. P. 2008, MNRAS, 387, 1627

Pandey, J. C., \& Singh, K. P. 2012, MNRAS, 419, 1219

Parker, E. N. 1988, ApJ, 330, 474

Priest, E. R., \& Forbes, T. G. 2002, A\&AR, 10, 313

Raassen, A. J. J., Mewe, R., Audard, M., \& Güdel, M. 2003, A\&A, 411, 509

Reale, F., Betta, R., Peres, G., Serio, S., \& McTiernan, J. 1997, A\&A, 325, 782

Reale, F., Güdel, M., Peres, G., \& Audard, M. 2004, A\&A, 416, 733

Robrade, J., Poppenhaeger, K., \& Schmitt, J. H. M. M. 2010, A\&A, 513, A12

Robrade, J., \& Schmitt, J. H. M. M. 2009, A\&A, 496, 229

Rutledge, R. E., Basri, G., Martín, E. L., \& Bildsten, L. 2000, ApJ, 538, L141

Sanz-Forcada, J., Favata, F., \& Micela, G. 2004, A\&A, 416, 281

Sasaki, M., Haberl, F., \& Pietsch, W. 2000, A\&AS, 143, 391

Schmitt, J. H. M. M., Fink, H., \& Harnden, F. R., Jr. 1987, ApJ, 322, 1023

Schmitt, J. H. M. M., Fleming, T. A., \& Giampapa, M. S. 1995, ApJ, 450, 392

Schmitt, J. H. M. M., \& Liefke, C. 2002, A\&A, 382, L9

Schmitt, J. H. M. M., \& Liefke, C. 2004, A\&A, 417, 651

Ségransan, D., Kervella, P., Forveille, T., \& Queloz, D. 2003, A\&A, 397, L5

Skrutskie, M. F., Cutri, R. M., Stiening, R., et al. 2006, AJ, 131, 1163

Stelzer, B., Schmitt, J. H. M. M., Micela, G., \& Liefke, C. 2006, A\&A, 460, L 35

Trenholme, D., Ramsay, G., \& Foley, C. 2004, MNRAS, 355, 1125

Tsikoudi, V., Kellett, B. J., \& Schmitt, J. H. M. M. 2000, MNRAS, 319, 1136

Tsuboi, Y., Imanishi, K., Koyama, K., Grosso, N., \& Montmerle, T. 2000, ApJ, 532, 1089

van den Oord, G. H. J., \& Mewe, R. 1989, A\&A, 213, 245

Wargelin, B. J., Kashyap, V. L., Drake, J. J., García-Alvarez, D., \& Ratzlaff, P. W. 2008, ApJ, 676, 610

Watson, M. G., Schrder, A. C., Fyfe, D., et al. 2009, A\&A, 493, 339

Welsh, B. Y., Wheatley, J. M., Seibert, M., et al. 2007, ApJS, 173, 673

White, S. M., Jackson, P. D., \& Kundu, M. R. 1989, ApJS, 71, 895 